\title{
Cambios en la edad de inicio del tratamiento de la dependencia alcohólica. ¿Debemos cambiar la oferta asistencial?
}

\author{
Monrás Arnau, M. \\ Psicólogo Adjunto. Unidad de Alcohología de la Generalitat de Catalunya. Hospital Clínico de Barcelona. Institut de Malalties Digestives.
}

\section{RESUMEN}

Introducción: Recientes datos epidemiológicos sobre el consumo de alcohol en España indican su importancia en los jóvenes, pero no es posible determinar si existe un aumento a través del tiempo o un empeoramiento de las consecuencias. Tampoco sabemos su repercusión en los inicios de tratamiento. Material y Método: Se analiza la edad de los nuevos casos de alcoholismo tratados en Catalunya en los últimos 10 años y en una unidad especializada en alcoholismo desde hace 25 años. Además se estudia la edad de los pacientes que utilizan las terapias grupales y la de los ingresos hospitalarios para desintoxicación. Resultados: La población atendida por su dependencia alcohólica es cada vez de mayor edad, tanto en Catalunya como en nuestra unidad especializada. La proporción de mujeres se mantiene estable. Paralelamente los ingresos y las terapias grupales se dirigen cada vez en mayor proporción a pacientes de mayor edad. Discusión: Aunque los presentes datos no pueden extrapolarse automáticamente a la población general, no se detecta un incremento del alcoholismo juvenil. Más bien está emergiendo un tipo de alcoholismo que podría etiquetarse como de "tercera edad". El uso de diversas técnicas terapéuticas parece adaptarse a los cambios generacionales, pero de forma mecánica, sin un conocimiento de su eficacia para cada grupo de edad. Se plantea la necesidad de diseñar instrumentos específicos y técnicas adaptadas a cada tipo de paciente alcohólico que ha ido identificándose con el tiempo.

Palabras clave: alcoholismo, distribución por edad, protocolos clínicos, centros de tratamiento de las drogodependencias, tipologías psicodiagnósticas.

\section{SUMMARY}

Introduction: Epidemiological data on alcohol consumption in Spain show the importance of alcohol consumption in the youths, but increase over time of this problem or a impairment of the consequences has not been yet studied. Either we know their repercussion in the treatment beginning. Material and Method: We analyzed the age of all the new cases of alcoholism treated in Catalonia (Spain) in the last 10 years as well as all the new patients treated in our alcohol dependence unit for the last 25 years. In addition we also studied the age of all patience that used group psychotherapies and those that required hospital detoxification. Results: Interesting enough, the population attended for alcohol dependency it is increasing on age, in all Catalonia as well as in our specific alcohol unit. The women ratio for the illness is maintained stable. At the same time, group psychotherapies and inpatient detoxification are consequently also increased on age. Discussion: The use of different therapeutic techniques seems to be adapted to the changes in time, but those changes are sometimes, made without a scientific evidence. It does not exist yet enough study about efficacy. Our data, show the need for instruments and specific techniques for each one of the types of alcoholic patients that are being identifying over time. This study suggest a growing tendency for a new group of alcoholic patients that may be named "third age".

Key words: Alcoholism, age distribution, clinical protocols, substance abuse treatment centers, psychodiagnostic typologies.

\section{INTRODUCCIÓN}

\section{1.- El consumo social de alcohol en los jóvenes.}

e unos años a esta parte la preocupación por la percepción social de que el consumo de drogas y alcohol aumenta entre los jóvenes está llevan- do a realizar cada vez más programas preventivos del uso de estas substancias dirigidos a este colectivo.

Parece claro que por una serie de razones de marketing son el blanco preferido de las campañas publicitarias de productos de alto consumo en general y de tabaco y bebidas alcohólicas (especialmente cerveza) en particular. 
Sin embargo creemos que no está tan claro en qué estado real se encuentra el consumo de alcohol en los últimos años y como puede haber evolucionado en el tiempo. Aún sabemos menos acerca de las consecuencias que tiene a medio y largo plazo entre los jóvenes, sobretodo en cuanto al riesgo de desarrollar un síndrome de dependencia al alcohol.

En la encuesta de la Generalitat de Catalunya de 1990 (1), el 9,7\% de jóvenes entre 15 y 29 años consumían más de $525 \mathrm{cc}$. de alcohol/semana frente a sólo un 7,1\% de la población general entre 15 y 64 años.

En esta encuesta se detectaba un aumento de los bebedores de riesgo entre jóvenes y adultos respecto de las encuestas de 1982 y 1986, siendo los jóvenes los que bebían más alcohol, por encima de la población general (170 vs. $142 \mathrm{cc} /$ semana), aunque a costa de un descenso en el consumo de vino y aumento de cerveza y destilados, patrón que parece general pero más acusado entre los jóvenes.

En 1994 un informe del Ministerio de Sanidad (2) concluía que los jóvenes se van incorporando más tempranamente al hábito de beber, con una edad media de inicio en el consumo de alcohol a los 15,5 años, especialmente los fines de semana, siendo el grupo de edad de 16 a 25 años el que consume más frecuentemente. Sin embargo esta edad parece variar según las fuentes ya que la encuesta sobre drogas a la población escolar de 1996 del Plan Nacional sobre Drogas (PND) señalaba que la edad media de inicio del consumo de alcohol parecía aumentar de los 13,4 años en 1994 a los 13,7 en 1996 (3), diferencia que tampoco permite conclusiones muy definitivas.

La encuesta de 1997 del PND en población general de mayores de 15 años (4) mostraba un aumento importante de la proporción de personas que habían consumido alcohol en el último año y mes, con respecto a la de 1995 y una ligera disminución en la proporción de consumidores diarios. La proporción de estos aumenta a medida que lo hace la edad del entrevistado, En cambio, la proporción de consumidores ocasionales es mayor en la población joven que en otros grupos de edad.

En la población de 15 a 65 años el 27,1\% bebe ocasionalmente, el 18,4\% 1-3 días a la semana y a diario o casi a diario el $18,4 \%$.

Sin embargo hay menos abstemios entre los jóvenes: de 14 a 18 años el 85,4\% había consumido alcohol los últimos 12 meses mientras sólo lo hacía un $63,8 \%$ de la población de 14 a 65 años (5).

EI PND (3) y la Generalitat de Catalunya (5) coinciden en que aumenta mucho con la edad, de los 14 a los 18 años, la proporción de bebedores y la intensidad del consumo de alcohol:

En 1994 la proporción de estudiantes consumidores de bebidas alcohólicas durante los 12 meses pre- vios a la encuesta aumentaba de un $67,3 \%$ a los 14 años al $86,9 \%$ a los 16 años, hasta un $92 \%$ a los 18 años. En 1996 también se produce el aumento con la edad; los porcentajes empiezan ligeramente más bajos y acaban mayores: $64,3 \%, 87 \%$ y $92,8 \%$ respectivamente, lo que lleva al PND a concluir que no se detecta un aumento de la proporción de consumidores de 1994 al 1996 ni cambios en las características del consumo.

Respecto a las características del consumo de alcohol entre los estudiantes se conoce bien que se produce fundamentalmente durante los fines de semana, siendo su intensidad esos días (viernes, sábado y domingo) entre 4 (hombres) y 8 veces (mujeres) más elevada que durante los días laborables.

Las encuestas mencionadas permiten concluir que los jóvenes beben de forma generalizada entre los 14 y 18 años. Respecto a los mayores lo hacen en menor frecuencia, pero en mayor cantidad total y con menos sujetos abstemios.

Estos datos, a pesar de su valor, presentan una serie de problemas para extraer una película clara de como ha evolucionado la situación: en primer lugar había existido hasta ahora poca sistematización en las encuestas, que eran una suma de acciones puntuales dispersas. Además tendían a realizarse en poblaciones territorial y socialmente distintas, con métodos y protocolos de recogida de información a veces incompatibles y en momentos temporales diferentes.

En los últimos años ha empezado a cambiar la situación, pero el breve intervalo de tiempo entre ellas hace que las diferencias sean pequeñas en muchos de los resultados y que no puedan considerarse significativas.

La explotación histórica de sus datos no será posible hasta dentro de un tiempo, por lo que de lo acontecido en los últimos 20 años en lugar de una película sólo disponemos de una serie de fotos fijas, con objetivos y filtros distintos y además enfocando hacia diferentes lados.

En la percepción social además se mezclan la información de los consumos con la de sus consecuencias: un ejemplo claro es el de los accidentes de tráfico. En ellos siempre se destaca la alta prevalencia de jóvenes entre los conductores y ocupantes accidentados, pero el aumento de la accidentabilidad en el tiempo (si es que antes no existía, ya que no se dispone de datos antiguos) puede deberse más a otros factores, como la mayor disponibilidad de dinero y vehículos o los cambios en las costumbres de consumo y los horarios de los establecimientos, que no a un posible aumento de los consumos alcohólicos en la juventud.

Las inferencias que podemos obtener de la información disponible no permiten determinar a ciencia 
cierta si el consumo de alcohol aumenta en según que intervalos de edad, o si se avanza la edad del primer consumo, aunque hay consenso en la existencia de un cambio en los patrones de uso, tendentes a un modelo anglosajón, de consumos concentrados en los fines de semana, mediante bebidas de mayor concentración alcohólica y utilización mezclada con otras substancias.

\section{2. - La aparición de problemas relacionados con el consumo de alcohol}

Si existe efectivamente un avance de la edad de inicio en el consumo de alcohol, o un aumento de las cantidades ingeridas en la juventud, es lógico esperar que aparecerán antes sus consecuencias.

Por ello y contando con una mayor detección precoz de estos problemas por parte de la red básica de salud, podemos suponer que también se traduzca en un aumento e inicio más temprano de los tratamientos de problemas relacionados con el alcohol entre personas jóvenes.

Hasta el momento no disponemos de datos acerca de la evolución en el tiempo de las características sociodemográficas de las personas que inician tratamientos por problemas relacionados con el alcohol.

\section{3.- El tratamiento de los problemas relacionados con el alcohol.}

Desde hace unos años se está haciendo hincapié en la necesidad de adecuar los tratamientos a las características específicas de cada tipo de enfermo (6).

Existen evidencias de que hay diferencias en el aprovechamiento de los tratamientos disponibles en función de la edad o el sexo del paciente. Algunas de las técnicas más utilizadas en el alcoholismo, como la terapia grupal, demuestran una menor efectividad entre pacientes jóvenes y mayor en las mujeres $(7,8$, 11). En el caso de los ingresos hospitalarios los jóvenes tienden a presentar menor sufrimiento psíquico, soporte familiar y motivación y abandonan antes el seguimiento post-alta, aunque pueden permanecer abstinentes durante largos periodos de tiempo (9).

La aparición del SIDA provocó una serie de cambios en las características de la población de pacientes heroinómanos, como un empeoramiento de su estado físico, un freno en la aparición de nuevos casos y un envejecimiento de la población atendida, que favorecieron la aparición de nuevos tratamientos de reducción de daños o de baja exigencia, con programas de intercambio de jeringuillas, de manteni- miento con metadona o con antagonistas opiáceos, etc.

Este ejemplo ilustra cómo debe adecuarse la oferta sanitaria al tipo de demanda de grupos poblacionales cambiantes. En el caso del alcoholismo corremos el riesgo de que la oferta de tratamientos quede desfasada al ir dirigida a subgrupos cada vez más reducidos del colectivo total de pacientes alcohólicos. Conocer cómo cambian estos es una necesidad ineludible si queremos ser eficaces con sectores cada vez más amplios de pacientes.

\section{4.- Objetivo:}

Se pretende estudiar si han habido en los últimos años en Catalunya cambios en la edad y sexo de los pacientes que solicitan tratamiento de su alcoholismo.

Secundariamente se estudiará si las técnicas empleadas se han ido adaptando a estos supuestos cambios.

Estos datos clínicos son una aproximación colateral y parcial, a partir sólo de las personas enfermas, pero puede ser útil para hacer inferencias indirectas acerca de las consecuencias del consumo de alcohol en la población general. También pueden servir para analizar en el tiempo la relación entre el consumo de alcohol y la aparición de consecuencias y la forma como se manifiestan estás mediante la petición de una ayuda terapéutica.

\section{MATERIAL}

El Organ Tècnic de Drogodependencies de la Generalitat de Catalunya centraliza los datos referidos a tratamientos de cualquier drogodependencia en la red de más de 50 centros públicos de Catalunya (X.A.D.- Xarxa d'Atenció a les Drogodependències). Dispone y publica desde hace más de 10 años datos estadísticos acerca de las características de los tratamientos y de los usuarios.

La Unidad de Alcohologia de la Generalitat de Catalunya en el Hospital Clínico de Barcelona (UA-HCB) sólo trata pacientes con criterios CIE-10 de abuso o dependencia al alcohol, aunque puedan existir también abuso y/o dependencia a otras drogas, como cocaína, cánnabis o BZD. Es uno de los centros de tratamiento del alcoholismo más antiguos de España.

A pesar del riesgo de falta de representatividad y de las limitaciones de fiabilidad que puede presentar estudiar un sólo centro, cuenta con dos ventajas para poder extrapolar tendencias temporales: 
a) Los pacientes. Dispone de una casuística larga y estable, de difícil consecución en otros ámbitos y anterior al inicio sistemático del tratamiento del alcoholismo en España. En 1991 realizó alrededor del 15\% de los tratamientos de nuevos casos de alcoholismo en Catalunya (10).

b) El equipo. El marco de trabajo ha estado sujeto a pocos vaivenes: más de 25 años ininterrumpidos de oferta asistencial que sólo se ha modificado para irse adaptando a la aparición de nuevas técnicas de tratamiento, un equipo profesional que ha tenido una continuidad en el tiempo y un encuadre, estilo terapéutico, lugar y ámbito de trabajo estables.

\section{MÉTODO}

1.- Se analiza la edad y sexo de los pacientes que inician tratamiento en todos los centros públicos de Catalunya desde 1991 (10). Esta limitación territorial se debe a la imposibilidad de disponer de datos a nivel de España acerca de los tratamientos del alcoholismo ya que el PND no ha contemplado hasta ahora el alcohol en sus publicaciones estadísticas.

2.- Posteriormente nos centraremos sólo en la UAHCB. Se han recuperado los historiales de los pacientes que iniciaron tratamiento por alcoholismo en la UA-HCB durante los años 1972, 1977, 1982, 1987, 1993 y 1997 para estudiar la evolución de su edad y distribución por sexo.

3.- Complementariamente también se analizan la edad y sexo de los pacientes de la UA-HCB a los que se indicaron dos técnicas específicas dentro del tratamiento: la terapia grupal desde 1988 y los ingresos hospitalarios para desintoxicación desde 1993. En estos se han excluido del análisis a los pacientes remitidos a Unidad de Desintoxicación Hospitalaria desde otros centros para realizar desintoxicación y que seguían tratamiento post-alta en el centro remitente.

\section{RESULTADOS}

\section{1.- Evolución del tratamiento en Catalunya.}

A nivel de Catalunya desde hace unos diez años se observa claramente (10), además de un paulatino incremento en el número total de personas tratadas por el alcohol, un ligero aumento en el porcentaje de personas mayores de 60 años, paralelo a la disminución de la frecuencia de menores de 30 años (Tabla 1). En nueve años se ha doblado el número de los primeros y disminuido en un $25 \%$ el de los segundos. En este tiempo la edad promedio ha pasado del rango entre 35 y 39 años a los 42 años de media.
La relación de hombres/mujeres de 4/1 es estable y se corresponde con la que siempre se ha considerado normal en los porcentajes de alcohólicos en tratamiento.

4.2.- Evolución de los inicios de tratamiento en la UA$H C B$.

a) Comparados los resultados anteriores con los de un centro específico como la UA-HCB se comprueba en primer lugar la práctica coincidencia en la edad media de inicio en 1997 (Tabla 2).

En el caso de la UA-HCB además se ha analizado separadamente en 1993 y 1997 a las personas visitadas a través de las interconsultas hospitalarias, que se caracterizan por una mayor edad. Este tipo de visita no se produce en casi ningún otro centro de tratamiento, por lo que no es en absoluto representativa del tipo de paciente visitado en los dispensarios públicos de drogodependencias de Catalunya.

b) De 1972 a 1987 tanto la edad media como su variabilidad tenían una gran estabilidad. En 1993 y 1997 en cambio se ha dado un salto cuantitativo y significativo, aumentando en casi 4 años la edad promedio para iniciar tratamiento alcohológico ambulatorio.

No es posible comparar los años 1972 a 1987 con la situación en el resto de centros de España al no existir datos, pero suponiendo que la UA-HCB fuera suficientemente representativa (lo que se da en el año 1997 comparada con Catalunya) podríamos extraer la conclusión de que la edad de inicio de tratamiento puede haber aumentado en general.

c) El porcentaje de hombres/mujeres sufre oscilaciones a partir de 1987 que no siguen un patrón fijo. Sin embargo siempre se mantiene un porcentaje de mujeres tratadas en la UA-HCB respecto de hombres superior al del resto de centros de Catalunya (que es de 1 a 4). Este es un dato histórico de la UA-HCB y conocido desde hace muchos años. Su explicación guarda relación con la sensibilidad hacia el alcoholismo femenino, del que ha existido un trabajo pionero por parte de todo el hospital (se mantiene la sobredetección relativa en las interconsultas hospitalarias).

\section{3.- Indicaciones de terapia grupal en la UA-HCB.}

Desde 1984 se han realizado más de 2000 inclusiones en terapia grupal (TG). No disponemos de los datos anteriores.

El análisis de los últimos 10 años revela claramente una progresiva disminución, dentro del total de pacientes a quienes se indica TG, en el número relati- 
TABLA 1:. Inicios de tratamiento por el alcohol en Catalunya

\begin{tabular}{|l|c|c|c|c|c|c|}
\hline $\begin{array}{l}\text { AÑO DE } \\
\text { INICIO }\end{array}$ & $\begin{array}{c}\text { Menores } \\
\text { de } 25 \text { años }\end{array}$ & $\begin{array}{c}\text { Menores } \\
\text { de } 30 \text { años }\end{array}$ & $\begin{array}{c}\text { Mayores } \\
\text { de } 60 \text { años }\end{array}$ & $\begin{array}{c}\text { Total de casos } \\
\text { (Todas las edades) }\end{array}$ & $\begin{array}{c}\text { Edad } \\
\text { promedio }\end{array}$ & SEXO \\
\hline 1991 & $207(7 \%)$ & $590(19,9 \%)$ & $200(6,8 \%)$ & 2962 & 35 a 39 & $79 \% / 21 \%$ \\
\hline 1992 & $192(5,4 \%)$ & $584(16,6 \%)$ & $227(6,5 \%)$ & 3505 & 35 a 39 & $82 / 18$ \\
\hline 1993 & $184(4,8 \%)$ & $555(14,5 \%)$ & $277(7,3 \%)$ & 3814 & $41,3 \pm 11$ & $80 / 20$ \\
\hline 1994 & $190(4,6 \%)$ & $606(14,8 \%)$ & $283(6,9 \%)$ & 4085 & $41,3 \pm 11$ & $81 / 19$ \\
\hline 1995 & $168(3,8 \%)$ & $613(14 \%)$ & $310(7,1 \%)$ & 4365 & 35 a 39 & $82 / 18$ \\
\hline 1996 & $151(3,4 \%)$ & $546(12,6 \%)$ & $302(7 \%)$ & 4318 & 35 a 39 & $81 / 19$ \\
\hline 1997 & $178(3,9 \%)$ & $557(12,3 \%)$ & $335(7,4 \%)$ & 4508 & 42 & $81 / 19$ \\
\hline 1998 & $156(3,2 \%)$ & $516(10,8 \%)$ & $400(8,4 \%)$ & 4760 & 42 & $81 \% / 19 \%$ \\
\hline
\end{tabular}

Fuente: Informes anuales desde 1991 hasta 1998. Sistema d'informació sobre drogodependències a Catalunya. Generalitat de Catalunya. Departament de Sanitat i Seguretat Social. Organ Tècnic de Drogodependències. 1991 a 1998, Barcelona.

TABLA 2: Porcentaje por sexos y edad de inicio del tratamiento alcohológico en la UA-HCB

\begin{tabular}{|c|c|c|c|}
\hline & $\mathrm{N}$ & Edad (media \pm S.D.) & Varones \% \\
\hline AÑO 1972 & 80 & $39 \pm 8,9$ & $71,3 \%$ \\
\hline AÑO 1977 & 197 & $39,4 \pm 12$ & $70 \%$ \\
\hline AÑO 1982 & 208 & $38,8 \pm 12$ & $69,5 \%$ \\
\hline AÑO 1987 & 246 & $39,3 \pm 10$ & $80,6 \%$ \\
\hline AÑO 1993 (Todos los pacientes) & 500 & $43,4 \pm 11$ & $74,4 \%$ \\
\hline AÑO 1993 (Sólo inicios ambulatorios) & 358 & $42,9 \pm 11$ & $75,9 \%$ \\
\hline AÑO 1993 (Sólo interconsultas hospitalarias) & 142 & $\begin{array}{c}44,7 \pm 11 \\
{ }^{*} t=1,5 ; p=n . s .\end{array}$ & $\begin{array}{c}73,2 \% \\
\text { ** Pearson= } 3,3 ; p=n . s .\end{array}$ \\
\hline AÑO 1997 (Todos los pacientes) & 502 & $44 \pm 11,6$ & $74,8 \%$ \\
\hline AÑO 1997. (Sólo inicios ambulatorios) & 332 & $43 \pm 10$ & $75 \%$ \\
\hline AÑO 1997. (Sólo interconsultas hospitalarias) & 170 & $\begin{array}{c}45,7 \pm 12 \\
{ }^{*} t=2,1 ; p=0,03\end{array}$ & $\begin{array}{c}73,5 \% \\
\text { ** Pearson= } 0,12 ; p=n . s .\end{array}$ \\
\hline \multirow[t]{2}{*}{ Comparación entre todos los años. } & & ANOVA & Ji cuadrado \\
\hline & & $F=13,3 ; p=0,000$ & Pearson= 10; $p=0,07$ \\
\hline
\end{tabular}

* Comparación (prueba t de Student) de la edad media entre pacientes ambulatorios e interconsultas.

* Comparación del porcentaje de hombres y mujeres (prueba de Ji cuadrado) entre pacientes ambulatorios e interconsultas.

vo de los menores de 30 años y un aumento de los mayores de 60 años (Tabla 3) e incluso de 65.

Comparado con los inicios de tratamiento en toda Catalunya se observa que la utilización de TG en pacientes jóvenes en la UA-HCB es siempre inferior porcentualmente al de casos que se tratan en ese intervalo de edad y que disminuye en paralelo con el porcentaje de pacientes jóvenes.

En el caso de pacientes mayores de 60 años se empezó con una utilización bajísima de la TG (el 1\% de todos los pacientes en TG en 1988) que aumentó paulatinamente $(4,8 \%$ de pacientes en $T G$ en 1991) aunque de forma inferior al porcentaje de pacientes mayores de 60 años tratados en Catalunya $(6,8 \%$ en 1991).

En la actualidad la situación es de utilizar la TG en un porcentaje de pacientes de tercera edad superior al del total que se tratan (en 1998 un 9,1\% de pacientes en TG en la UA-HCB tienen más de 60 años, mientras que sólo representan un $8,4 \%$ de los pacientes que inician tratamiento).

El porcentaje de mujeres en TG siempre se ha mantenido alrededor del 30\%, el cual es superior al de las que realizan tratamiento en total. 
TABLA 3: Edad de inclusión en terapia grupal en la UA-HCB

\begin{tabular}{|l|c|c|c|c|c|c|}
\hline AÑO DE INCLUSION & $\begin{array}{c}\text { Menores } \\
\text { de } 25 \text { años }\end{array}$ & $\begin{array}{c}\text { Menores } \\
\text { de } 30 \text { años }\end{array}$ & $\begin{array}{c}\text { Mayores } \\
\text { de } 60 \text { años }\end{array}$ & $\begin{array}{c}\text { Mayores } \\
\text { de } 65 a n ̃ o s\end{array}$ & $\begin{array}{c}\text { SEXO } \\
\text { Varones \% }\end{array}$ & $\begin{array}{c}\text { Total (Todas } \\
\text { las edades) }\end{array}$ \\
\hline 1988 & 8 & $32(15 \%)$ & $2(1 \%)$ & 0 & $72 \%$ & 213 \\
\hline 1989 & 3 & $20(11,4 \%)$ & $6(3,4 \%)$ & $4(2,2 \%)$ & $78 \%$ & 175 \\
\hline 1990 & 5 & $18(12 \%)$ & $7(4 \%)$ & $3(2 \%)$ & $74 \%$ & 149 \\
\hline 1991 & 5 & $20(16,1 \%)$ & $6(4,8 \%)$ & $2(1,6 \%)$ & $71 \%$ & 124 \\
\hline 1992 & 1 & $12(6,9 \%)$ & $2(1,1 \%)$ & $1(0,5 \%)$ & $69 \%$ & 173 \\
\hline 1993 & 2 & $8(6 \%)$ & $4(3 \%)$ & 0 & $68 \%$ & 133 \\
\hline 1994 & 2 & $9(11,1 \%)$ & $1(1,2 \%)$ & 0 & $70 \%$ & 81 \\
\hline 1995 & $1(0,8 \%)$ & $5(4 \%)$ & $5(4 \%)$ & 0 & $70 \%$ & 125 \\
\hline 1996 & 0 & $8(6,9 \%)$ & $1(0,8 \%)$ & 0 & $69 \%$ & 115 \\
\hline 1997 & $1(0,8 \%)$ & $5(4,3 \%)$ & $6(5,1 \%)$ & $2(1,7 \%)$ & $74 \%$ & 116 \\
\hline 1998 & 0 & $6(5,5 \%)$ & $10(9,1 \%)$ & $5(4,6 \%)$ & $75 \%$ & 109 \\
\hline 1999 (Hasta 1 de Noviembre) & 2 & $5(4,6 \%)$ & $11(10,1 \%)$ & $5(4,6 \%)$ & $68 \%$ & 108 \\
\hline Total (desde 1990) & 19 & $96(7,7 \%)$ & $53(4,2 \%)$ & 18 & & 1233 \\
\hline
\end{tabular}

\section{4.- Ingresos para desintoxicación en la UA-HCB.}

Aunque en los últimos 4 años se ha cambiado de Sala (de Psiquiatría a Medicina Interna) y se ha acortado la duración (de 4 a 1 semanas), aumentando el número de los ingresos (entre los que no contamos a los pacientes remitidos desde otros centros), no ha sido así en los criterios de su indicación por la UA$\mathrm{HCB}$, que se han mantenido dirigidos a pacientes con dificultades para realizar la desintoxicación ambulatoria, sea por falta de soporte socio-familiar, patología física o psíquica, dependencia conjunta a BZD o tratamientos ambulatorios previos fracasados.

En sólo 4 años (de 1993 a 1997) se ha operado un cambio espectacular en la edad de los pacientes ingresados para desintoxicación alcohólica, disminuyendo los menores de 30 años del 33\% en 1993 al $4,3 \%$ en 1998. La otra cara de la moneda es el aumento mucho menos pronunciado de los mayores de 60 años, que pasan del 5,4\% al 7,8\% en el mismo periodo (Tabla 4).
Los cambios en las frecuencias de cada sexo no tienen ninguna significación, pues están mediatizados e igualados artificialmente por las necesidades de organización de la Sala para disponer de camas en habitaciones separadas.

\section{DISCUSIÓN}

La limitación del tipo de datos disponible no permite extrapolaciones a la población general, pero sí que nos da una película fiable de la evolución de los inicios de tratamiento por parte de una población clínica, que sí puede considerarse representativa de una amplia población de pacientes alcohólicos españoles.

Estos datos claramente no apoyan la percepción de un aumento de los casos de alcoholismo juvenil (antes de los 30 años). Por el contrario todo indica la aparición de un fenómeno de progresivo envejecimiento de los pacientes alcohólicos tratados, que llegan incluso a edades relativamente avanzadas

TABLA 4: Ingresos en Sala de la UA-HCB para desintoxicación

\begin{tabular}{|l|c|c|c|c|c|c|}
\hline & $\begin{array}{c}\text { Menores } \\
\text { de 25 años }\end{array}$ & $\begin{array}{c}\text { Menores. } \\
\text { de 30 años }\end{array}$ & $\begin{array}{c}\text { Mayores. } \\
\text { de } 60 \text { años }\end{array}$ & $\begin{array}{c}\text { Mayores. } \\
\text { de 65años }\end{array}$ & $\begin{array}{c}\text { SEXO } \\
\text { Varones }\end{array}$ & $\begin{array}{c}\text { Total (Todas } \\
\text { las edades). }\end{array}$ \\
\hline 1993 & 7 & $30(33 \%)$ & $5(5,4 \%)$ & 1 & $57 \%$ & 91 \\
\hline $1997:$ & 2 & $9(3,5 \%)$ & $14(5 \%)$ & 5 & $74 \%$ & 254 \\
\hline $1998:$ & 5 & $11(4,3 \%)$ & $20(7,8 \%)$ & 10 & $67 \%$ & 255 \\
\hline Total $97-98$ & 7 & $20(3,9 \%)$ & $34(6,6 \%)$ & 15 & & 510 \\
\hline
\end{tabular}


(mayores de 65 años). En cuanto a la proporción de mujeres tratadas no se detecta la existencia de cambios.

Los resultados sugieren varias posibles explicaciones no excluyentes:

En primer lugar es probable que exista, más que una detección más precoz, una mayor detección global del consumo perjudicial del alcohol, del alcoholismo y de sus consecuencias. El envejecimiento de los nuevos enfermos tratados puede estar causado en gran parte por la detección de las patologías orgánicas relacionadas con el alcohol, por parte de los servicios de salud (atención primaria y hospitalarios), que, como muestran las interconsultas hospitalarias, se producen en personas de mayor edad.

Otro factor claro es el envejecimiento poblacional de las sociedades avanzadas, por lo que no debería sorprendernos el envejecimiento de los pacientes de muchas patologías, especialmente las de evolución crónica.

Por supuesto también es posible un avance de la edad de inicio del consumo de alcohol en los últimos tiempos que no se detectaría aún debido al "silencio clínico" de unos 10 años hasta que se manifiesta el alcoholismo. Sin embargo el cambio del patrón juvenil de consumo hacia un estilo anglosajón de fines de semana, con intoxicaciones más que problemas orgánicos crónicos, favorecería mas bien la aparición precoz de trastornos conductuales agudos. De esta forma estos casos también serian detectados por los servicios de salud sobretodo a través de las urgencias. El servicio de urgencias de nuestro hospital nos remite posteriormente estos pacientes (aunque no tengan dependencia alcohólica y sólo exista un abuso), pero hasta el presente tampoco hemos detectado un aumento de estos casos.

La mezcla del alcohol con otras substancias puede ser causa de un perfil distinto de consumidor, con unos problemas de características y evolución distintas y que hagan uso de otros tipos de recursos asistenciales (sanitarios o no) y en otros momentos. Por ello es básico disponer de los datos integrados de los tratamientos de todas las drogodependencias (no separando el alcohol de las ilegales como hasta ahora), para conocer si puede existir un trasvase de los drogodependientes de una substancia a otra con los años, especialmente a partir del alcohol.

Una explicación secundaria a ésta seria un posible menor acceso de los jóvenes a los tratamientos, pero no hay ningún dato ni factor que conozcamos pudiera causarlo.

Finalmente, y aunque parece prematuro, no podemos descartar que empiecen a dar sus primeros frutos los programas preventivos iniciados en los últimos 10 años y que se traduzcan en cambios observables de los hábitos de la población. Este hecho iría en con- tra del alarmismo que en algunos momentos aparece en medios de comunicación y reforzaría la necesidad y utilidad de estos programas preventivos.

Una vez analizada la situación de los consumidores otra cosa es el tipo de respuestas terapéuticas que ofertamos: es curioso en el caso de la UA-HCB que la adaptación a la nueva situación (de la que no éramos plenamente conscientes hasta este estudio) ha pasado por ofrecer a personas de mayor edad técnicas que antes destinábamos a poblaciones jóvenes, como los grupos de terapia, sin una autocrítica de su efectividad.

De todas maneras no debe sorprendernos pues usamos demasiadas técnicas basándonos en la repetición, la experiencia personal y las buenas intenciones más que en las evidencias científicas: actualmente y de forma paulatina vamos encontrándonos casos de tratamientos en que una revisión y análisis crítico de ellos nos revela su escasa eficacia, o que los destinamos a personas que no son las idóneas. Ello puede estarnos pasando con la utilización de grupos de terapia para jóvenes $(8,11)$ y corremos el riesgo de que vuelva a ocurrirnos dentro de 5-10 años en caso de que el tipo de TG que usamos se nos muestre poco útil en los pacientes mayores de 60 años. El mismo razonamiento se puede emplear en el uso dado hasta ahora de fármacos tipo disulfiran, de los controles de orina o de otros psicofármacos de utilidad dudosa.

En cuanto al incremento en la utilización de ingresos para desintoxicación de pacientes de edad más avanzada puede obedecer, entre otras razones, a su peor estado físico-psíquico, a una mayor probabilidad de dependencia asociada a BZD y a una acumulación de más tratamientos fracasados.

Los jóvenes alcohólicos han disminuido en porcentaje sobre los pacientes tratados, pero existe la posibilidad, que debería estudiarse, de que hayan modificado sus características. Entre estos cambios se hallaría un mayor uso conjunto de otras substancias y mayor frecuencia de psicopatología, con lo que quizás utilicen ahora más los servicios de otras drogodependencias y/o hospitalarios de salud mental. Por otra parte el interés de los equipos de salud mental (especialmente de psiquiatría), y quizás su oferta de servicios hacia las drogodependencias, ha aumentado en los últimos años.

A fin de cuentas, y lamentablemente, es habitual adaptar la demanda de los pacientes a la oferta de tratamientos disponibles y no al revés, con lo que ante las situaciones nuevas primero vamos probando remedios viejos. Es muy posible que debamos replantearnos (como en el caso de los heroinómanos) a corto plazo las estrategias de tratamiento, para poder atender correctamente a los diversos subgrupos de alcohólicos que van emergiendo con el tiempo: 
-Una proporción cada vez relativamente menor de pacientes jóvenes con poca dependencia y patrones de abuso o consumo compulsivo, que pueden presentar comorbilidad psiquiátrica y que se benefician poco de los tratamientos orientados a la abstinencia total.

-Un progresivo aumento en la proporción de pacientes ancianos que antes no se detectaban, que tienen un mayor riesgo de dependencia combinada a BZD (muchas veces yatrogénica), pero que al mismo tiempo están más conservados psíquica y físicamente gracias a la mayor detección y cuidados sanitarios.

-Un número importante, acumulativo y cada vez de mayor edad, de pacientes alcohólicos cronificados (9), con deterioro social y psicológico importante que no consiguen estabilizar un mínimo su abstinencia a pesar de seguir sucesivos tratamientos,

-Pacientes orgánicamente muy graves (por ejemplo candidatos a trasplante hepático) que anteriormente no llegaban a los programas de tratamiento del alcoholismo.

No basta con esforzarnos en imaginar nuevas estrategias terapéuticas y en ser flexibles en adaptarlas a la realidad cambiante. También hemos de ser rápidos en detectar estos cambios para no llegar tarde.

\section{BIBLIOGRAFIA}

1. Òrgan Tècnic de Drogodependències. Enquesta sobre consum i actituds respecte del tabac, l'alcohol i les altres drogues a Catalunya. Any 1990. Generalitat de Catalunya, 1990.

2. Vargas F, Robledo T, Espiga I. Alcohol y salud pública. Serie de informes técnicos, $n^{\circ} 1$. Secretaria General de Salud. Dirección General de Salud Pública. Ministerio de Sanidad y Consumo, Madrid, 1994.

3. Plan Nacional sobre Drogas. Encuesta sobre Drogas a la Población Escolar 1994 y 1996. Ministerio del Interior. Página web del Observatorio Español. Madrid, 1996.

4. Plan Nacional sobre Drogas. Encuesta domiciliaria sobre uso de drogas 1995. Ministerio del Interior. Página web del Observatorio Español. Madrid, 1995

5. Òrgan Tècnic de Drogodependències. Enquesta escolar sobre drogues. Any 1994. Generalitat de Catalunya, 1994.

6. Project Match Research Group. Matching Alcoholism Treatments to Client Heterogeneity: Project MATCH Posttreatment Drinking Outcomes. J Stud Alcohol 1997; 58(1): 7-29.

7. Monras M, Freixa N, Martinez M, Bach L. Abandono de la terapia grupal en pacientes alcohólicos. Seguimiento de diez grupos. Adicciones 1990; 2(2): 93-104.

8. Monras M, Gual A. Attrition in group therapy with alcoholics: a survival analysis. Drug and Alcohol Review 2000; 19; 55-63.

9. Monras M, Garcia S, Torres M, Gual A, Ortega L. El ingreso hospitalario en el tratamiento de los alcoholicos cronificados. Adicciones 1998; 10(2): 151-160.

10. Informes anuales (desde 1991 hasta 1998). Sistema d'informació sobre drogodependencies a Catalunya. Generalitat de Catalunya. Departament de Sanitat i Seguretat Social. Organ Tècnic de Drogodependències. 1991 a 1998, Barcelona.

11. Monras M. Indicaciones de terapia grupal para alcohólicos. Actas Españolas de Psiquiatría 2000; (en prensa). 